\title{
BMJ Open Assessing the chronic respiratory health risk associated with inhalation exposure to powdered toner for printing in actual working conditions: a cohort study on occupationally exposed workers over 10 years
}

Toshio Nakadate, Yuko Yamano, Takenori Yamauchi, Shigeko Okubo, Daichi Nagashima

To cite: Nakadate T, Yamano $Y$, Yamauchi T, et al. Assessing the chronic respiratory health risk associated with inhalation exposure to powdered toner for printing in actual working conditions: a cohort study on occupationally exposed workers over 10 years. BMJ Open 2018;8:e022049. doi:10.1136/ bmjopen-2018-022049

- Prepublication history for this paper is available online. To view these files, please visit the journal online (http://dx.doi. org/10.1136/bmjopen-2018022049).

Received 1 February 2018 Revised 19 August 2018 Accepted 20 September 2018

Check for updates

(c) Author(s) (or their employer(s)) 2018. Re-use permitted under CC BY-NC. No commercial re-use. See rights and permissions. Published by BMJ.

Department of Hygiene, Public Health, and Preventive Medicine, Showa University School of Medicine, Tokyo, Japan

Correspondence to

Professor Toshio Nakadate;

nakadate@med.showa-u.ac.jp

\section{ABSTRACT}

Background Little epidemiological evidence exists regarding the chronic respiratory effects of inhaled powdered toner exposure in humans, although several case reports have suggested the existence of lung disorders that might be related to exposure to toner dust. Objective We aimed to estimate the chronic health risk to humans associated with routine toner dust exposure in copier industry workers under current actual work conditions.

Design A prospective observational cohort study of occupational population.

Methods Changes in chest radiogram, spirometry measurements and serum and urine biomarkers of biomedical responses to extrinsic stress, as well as subjective symptoms were longitudinally observed for up to 10 years in Japanese copier industry workers responsible for the manufacturing, maintenance or recycling of powdered toner or toner-using machines. A total of 694 subjects who did not change their work category during the follow-up and were free from chronic respiratory diseases at the baseline survey provided reliable results on at least three survey occasions during 3 years or more of follow-up.

Results Typical fibrosis findings associated with pneumoconiosis was not observed on chest radiograms. No significant differences associated with toner exposure were noted in the frequency of new incidence of either non-specific findings on chest radiogram or serum fibrosis biomarkers (sialylated carbohydrate antigen KL-6 and surfactant protein D). However, the exposed subjects tended to show increases in the frequency of respiratory symptoms and reduced spirometry results during the follow-up compared with the control group, although significant differences were only seen in chronic cough. Conclusions Under the current reasonably controlled work environmental conditions, lung fibrotic changes caused by inhaled dust exposure, including powdered toner, appear to be relatively uncommon; however, nonspecific temporal irritation causing subjective symptoms and inflammatory responses might exist.
Strengths and limitations of this study

- An occupational cohort of workers in business machine industry exposed to printing toner dust in various types of work was followed for up to 10 years regarding their respiratory health status on an annual basis, mainly focusing on lung fibrotic changes.

- The incidence of newly emerging lung fibrotic changes was compared between the exposed workers and the non-exposed controls using wide-range health outcomes including chest $X$-ray findings, lung function results and serum and urine biomarkers, as well as subjective symptoms.

- The total dust exposure during toner handling was measured in a representative sample of workers, but the fraction of toner particles in the whole dust exposure cannot be estimated accurately.

- Conventional chest radiography was used to evaluate lung fibrotic changes in an epidemiological research setting; however, CT might have been more sensitive to early minimal fibrotic changes.

- Healthy worker bias might exist because some portion of the cohort ceased participation during the follow-up due to resigning, retiring or transferring, although no toner-related health problems, such as lung fibrosis, were reported among these subjects.

\section{INTRODUCTION}

Possible harmful effects of inhaled photocopier toner dust have been a matter of concern since the publication of several case reports suggested the existence of toner-related pulmonary disorders. ${ }^{1-3}$ However, little evidence is available regarding the potential health risk associated with toner dust inhalation in occupational and general home environments where photocopier machines and laser printing devices are in use. 
We reported the results of a cross-sectional study of toner-handling workers engaged in several types of toner exposure work. ${ }^{4}$ Although the results suggested the limited possibility of adverse effects due to toner dust inhalation, it was also suggested that a study with longitudinal design would be useful for a more detailed examination of the hypothesised association between toner dust exposure and adverse health effects under current actual work conditions.

We therefore conducted a longitudinal study of toner-handling workers for 10 years in a business machine-producing company and affiliated companies involved in maintaining and recycling these machines in order to obtain longitudinal evidence to determine a possible health risk associated with a practical level of toner dust exposure under current actual work conditions, with particular focus on chronic fibrotic changes in the lungs.

This is the first report of this study and mainly presents the results of analyses of the possible chronic health effects, including lung fibrotic changes as the principal research target, associated with toner dust exposure under current actual work conditions based on longitudinally observed health data.

\section{METHODS}

\section{Subjects}

The subjects were employees of seven branches of a group of companies that produced, maintained and recycled photocopiers, laser printers and related products for printing with the use of powdered toner. We identified four categories of toner-handling work among the subjects. Toner production (TPD) represents work associated with any process in the course to producing powdered toner, such as mixing and moulding raw materials, crushing toner mass and filling and packing the products. Designing and development of machines (DDMs) represents work associated with developing new models of toner-using business machines, in which various types of toner-handling procedures are involved. Maintenance (MTN) represents the work associated with maintaining photocopiers and laser printers in users' offices and homes. Recycling (RCL) represents work associated with processing recycled toner cartridges. The air environment was generally well maintained using local ventilation instruments according to the regulations in Japan. The workers were therefore encouraged to wear respiratory protection masks in only limited situations where heavy exposure might occur, such as when cleaning the mixing tanks or performing maintenance on/repairing equipment.

The survey on the health status of the subjects started in the fall of 2003 and was repeated until 2013 basically on an annual basis in the same manner as the first one in 2003. Throughout the entire study period, a total of 1176 toner handling workers (587 for TPD, 207 for DDM, 346 for MTN and 36 for RCL) participated in at least one of these

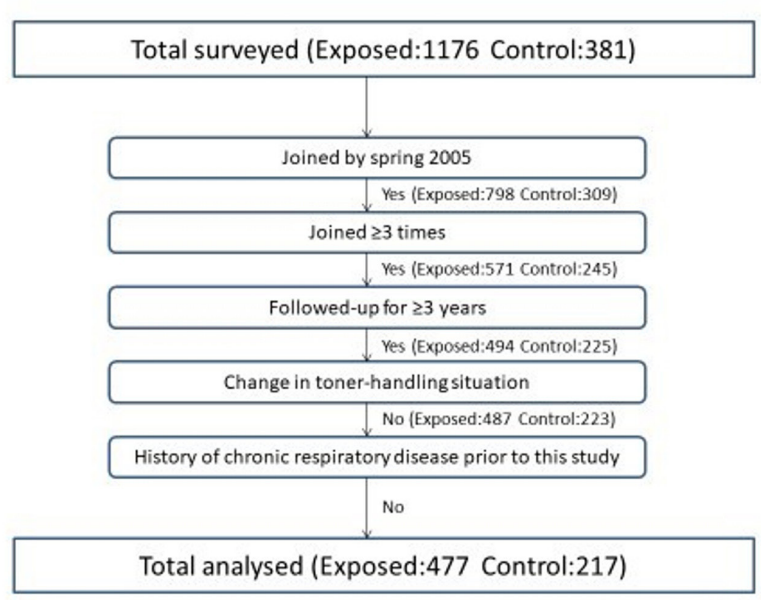

Figure 1 The number of subjects analysed based on the inclusion criteria of this study.

surveys. However, some participants ceased participation during an early stage of the study for various reasons other than toner-related health problems (eg, resigning, retiring or transferring) and others started participating in a later stage of the study. Therefore, we defined the subjects with a sufficient follow-up duration for a longitudinal data analysis in this paper as those who started participating by the spring of 2005 and were followed-up for 3 years or more with at least three surveys throughout the entire study period. In addition, subjects were those who had not changed their work category (concerning the presence or absence of toner handling) during their follow-up. The flow chart in figure 1 shows the number of participants included in or excluded from the analysis based on the above-mentioned criteria.

The number of toner workers who met the above criteria was 487. As a reference population for comparison with these toner-handling workers, an additional 309 control subjects who had never participated in any types of toner-handling work described above also participated in this study by the spring of 2005. Among them, the 223 who met the same criteria as exposed workers stated above were analysed. Control subjects were recruited at each participating branch and amounted to roughly half the number of toner-handling workers. The control subjects basically worked in different buildings from the ones where the exposed workers were engaged in toner-handling work. While the control subjects occasionally happened to enter the factory area, such accidental exposure was quite limited.

\section{Health status indices}

Subjects' health status was evaluated on an annual basis from various health-related aspects using physiological, biochemical and radiographic examination tools as well as via the assessment of subjective symptoms and clinical signs. This evaluation focused mainly on chronic respiratory deterioration, including pulmonary fibrotic conditions, and biological responses to inhaled potentially 
harmful substances. Standardised methods were used for health status measurement to keep the measurement bias as small as possible. The biomedical indices examined in this study are described below.

\section{Chest X-ray findings}

Chest radiography was carried out to obtain a standardised anterior-posterior X-ray film by using prescribed standard procedures to detect mineral dust pneumoconiosis in Japan. ${ }^{5}$ Each X-ray film was read by one of three readers with sufficient experience in the radiographic diagnosis of pneumoconiosis while referencing the standard films for the diagnosis. ${ }^{6}$ The readers were privy to no information regarding the exposure status of the subjects. The severity of fibrotic findings in the lung fields was classified into 12 grades, from $0 /-$ to $3 /+$, according to the distribution and density of the small opacities. This classification was compatible with that of the International Labor Organization. ${ }^{7}$ However, we noted none of the fibrotic findings typically observed in mineral dust pneumoconiosis cases with $1 / 1$ or a more severe grade profusion during early surveys. Therefore, we additionally recorded mild, non-specific findings, such as sparse irregular opacity, unclear interstitial shadow or disrupted vascular shadow in radiographs. These findings were potentially related to early effects of toner toxicity and might be caused by acute or subacute non-specific irritation or infection, which might be reversible.

\section{Questionnaire survey}

Detailed information regarding respiratory symptoms, allergic symptoms, past and present medical history and lifetime smoking history was obtained using a translated version of a self-administered questionnaire standardised by the American Thoracic Society (ATS-DLD-78A), ${ }^{8}$ with slight modification and several additional questions on allergies of the eye, nose and skin. The responses were certified by several trained interviewers, and supplemental interviews were held when necessary. Subjects were considered to have a symptom when they gave an affirmative response to questions about the specific symptom. The lifetime work history was also confirmed during this interview.

\section{Spirometry}

Subjects were asked to repeat the forced expiratory manoeuvre up to a maximum of five times in the standing position in order to obtain acceptable and reproducible spirometry results. The mechanical specifications of the spirometer used (DISCOM-21F $\times 2$; CHEST Co Ltd, Tokyo, Japan) met the standards stipulated by ATS. ${ }^{9}$ Routine BTPS correction and back-extrapolation were carried out. The calibration of the spirometer, spirometry measurements and evaluation of the results were based on prescribed procedures described in detail elsewhere. ${ }^{10}$

The percent predicted values standardised on sex, age and height were calculated for forced vital capacity (FVC), forced expiratory volume in $1 \mathrm{~s}$ (FEV1) and FEV1/FVC in each subject based on the prediction equations published by the Japanese Respiratory Society. ${ }^{11}$ The values of FVC, FEV1 and FEV1/FVC were defined as reduced when they were smaller than the lower value of the CI of the authorised reference equations described above. All figures were calculated from the best manoeuvre yielding the largest sum of FVC and FEV1 for each subject. ${ }^{12}$

For the analysis of the longitudinal changes in spirometry indices, we calculated the height-squared proportional values of FVC $\left(\mathrm{FVC} / \mathrm{HT}^{2}\right)$ and FEV1 $\left(\mathrm{FEV} 1 / \mathrm{HT}^{2}\right)$ to adjust for differences in the body size. The annual decline in $\mathrm{FVC} / \mathrm{HT}^{2}$ and $\mathrm{FEV} 1 / \mathrm{HT}^{2}$ were calculated for each subject as a regression coefficient using a simple linear regression equation of those values against age during the follow-up period.

\section{Serum and urine biomarkers}

Peripheral venous blood and urine $(10 \mathrm{~mL}$ and $20 \mathrm{~mL}$, respectively) were obtained from each subject at the time of every survey. Serum was immediately separated from whole blood sample using a routine procedure. Then, serum and urine samples were deeply frozen at $-80^{\circ} \mathrm{C}$ until analyses were carried out.

As the indices of biological responses to inhaled extrinsic irritants like toner particles, we employed the following biomarkers: highly sensitive $\mathrm{C}$ reactive protein (CRP), non-specific immunoglobulin E (IgE), sialylated carbohydrate antigen KL-6 (KL6) and lung surfactant protein D (SPD) in serum, and 8-hydrovy deoxy guanosine $(8 \mathrm{OHdG})$ in urine. In Japan, serum CRP, IgE, KL6, and SPD levels are routine clinical laboratory test items assessed in common medical practice. The analyses of those results were therefore carried out according to the appropriate standard operation procedures in SRL Inc, Tokyo., Japan, which has been providing high-quality testing under the certification of the Japan Accreditation Board, a public service juridical foundation for ensuring the reliability of clinical laboratory testing in Japan.

Urine $8 \mathrm{OHdG}$ was measured using an ELISA kit (New 8OHdG Check ELISA Kit; Japan Institute for the Control of Aging) and was adjusted for urine condensation using the urine creatinine concentration as the denominator.

The result of the measurement of each index was judged to be out of the normal range when the value exceeded the following figures: $1500 \mathrm{ng} / \mathrm{mL}$ for CRP, 173 $\mathrm{IU} / \mathrm{mL}$ for IgE, $500 \mathrm{U} / \mathrm{mL}$ for KL6, $110 \mathrm{ng} / \mathrm{mL}$ for SPD and $20 \mathrm{ng} / \mathrm{mg}$ creatinine for $8 \mathrm{OHdG}$.

\section{Exposure assessment}

We directly measured the personal exposure levels in individual toner-handling workers.

The average diameter of the toner particles contained in the products handled by the workers in this study was about $10 \mu \mathrm{m}$. Workers were asked to wear a portable personal dust sampler (NWPS-254 sampler with a T60A20 sampling filter, SIBATA SCIENTIFIC TECHNOLOGY Ltd, Tokyo, Japan) that continuously sampled the air in the workplace environment at $2.5 \mathrm{~L} / \mathrm{min}$ air flow driven 
by a MP-2N or MP-3 $\Sigma$ pump (SIBATA SCIENTIFIC TECHNOLOGYLtd). The sampling head was located around the neck of each subject to collect particles suspended in the air throughout a given period of toner-related work. The respirable fraction was separated using filters designed to cut $50 \%$ of $4 \mu \mathrm{m}$ diameter particles. The concentration of total and respirable dust exposed to the workers was calculated as a time-weighted average value for 8 hours (TWA-8h) based on the amount of dust collected on the filter and the total volume of air inspired by the sampling pump. Measurements were conducted basically twice a year for at least five workers in each toner-handling procedure, yielding a total of 2042 measurements (1751 for TPD, 81 for DDM, 88 for MTN and 122 for RCL) throughout the study period. Those values obtained were evaluated with reference to the recommendations by the Japan Association of Industrial Health (JAIH) ${ }^{13}$ We also analysed several chemical components that were generated when the toner was heated at a temperature of around $180^{\circ} \mathrm{C}$ as representative components of powdered toner using gas chromatography and mass spectrometry in order to estimate the toner exposure separately from other types of dusts suspended in the air. However, we were unable to obtain reliable estimates of the amount of toner dust fraction in the whole dust cluster collected on the sampling filter, mainly because of large variability of the values depending on the types of toner handled.

\section{Analyses}

Cross-sectional evaluation of baseline data

For each subject, we considered the results obtained in the first year of participation as the baseline data, indicating the condition at the beginning of the longitudinal observation of this study. The relative frequency of subjects with positive results was calculated for each health-related item described above (prevalence) and compared statistically between the exposed subjects as a whole and the control subjects. The significance of differences in the prevalence between the exposed subjects and controls was assessed using a $\chi^{2}$ test and Fisher's exact test as appropriate. A multiple logistic regression analysis was used to obtain the OR estimates adjusted for possible confounding factors.

\section{Longitudinal evaluation of chronic fibrotic findings}

For the longitudinal analysis, we mainly focused on the results associated with chronic respiratory toxicity, including the fibrotic potential of toner dust inhalation exposure. To this end, we analysed the changes in chest radiographic findings during the follow-up period. We also used three pulmonary function indices (FVC, FEV1 and FEV1/FVC) and two serum biomarkers (KL6 and SPD, both of which are recognised as useful markers of active-phase fibrotic conditions in the lungs). ${ }^{14}$ In addition, we selected three chronic respiratory symptoms associated with chronic respiratory diseases, such as fibrotic lung changes, namely chronic cough, chronic phlegm and breathlessness. Chronic cough, as well as chronic phlegm, was considered to be present when the symptom existed for 3 months or more in a year. Breathlessness was considered to be present when a subject walked slower than their counterparts of the same age on level ground.

In the longitudinal data analysis, we evaluated the frequency of abnormalities that newly emerged during the follow-up observation (incidence). Abnormalities were considered to be present when the respiratory symptom or the X-ray findings were newly noted or when the pulmonary function or biomarker values exceeded the normal range during follow-up. Therefore, those subjects who already had abnormalities at their baseline survey were excluded from the longitudinal data analysis. The incidence was compared between the exposed workers as a whole and the control subjects, as well as by the work categories. In a similar manner to the cross-sectional analyses, the incidence was compared using a $\chi^{2}$ test or Fisher's exact test as appropriate, and the OR estimates adjusted for confounders were calculated using a multiple logistic regression analysis.

\section{Statistical analyses}

For comparisons by exposure categories, the $\chi^{2}$ test and Fisher's exact test as well as a multiple logistic regression analysis were performed for categorical variables, and a simple t-test and an analysis of covariance were performed for numeric variables, with the alpha error level set at 0.05 . All statistical tests and estimations were carried out using the SAS statistical package on a personal computer (PC-SAS V.9.2).

\section{Ethical considerations}

The objectives and outline of the study were explained to the subjects at the beginning of the study, and written informed consent was obtained from each participant. All information obtained in this study was handled in accordance with the guidelines for epidemiological studies authorised in Japan. ${ }^{15}$

\section{Patient involvement}

Patients were not involved in this study.

\section{RESULTS}

As stated earlier, we selected 710 workers (489 exposed and 221 controls) as the subjects for this longitudinal data analysis. Among them, 16 subjects reported that they had suffered from chronic respiratory diseases prior to the beginning of this study (chronic obstructive pulmonary disease and/or pulmonary tuberculosis). These subjects were therefore excluded, and the remaining 694 subjects were ultimately analysed (figure 1 ). The average number of surveys in which those subjects participated was 6.8 , and the average follow-up duration (elapsed time from the first observation to the final one) was 7.4 years. No significant difference was observed in those average values between the exposed and control groups. 
Table 1 Background characteristics of the subjects according to the exposure category

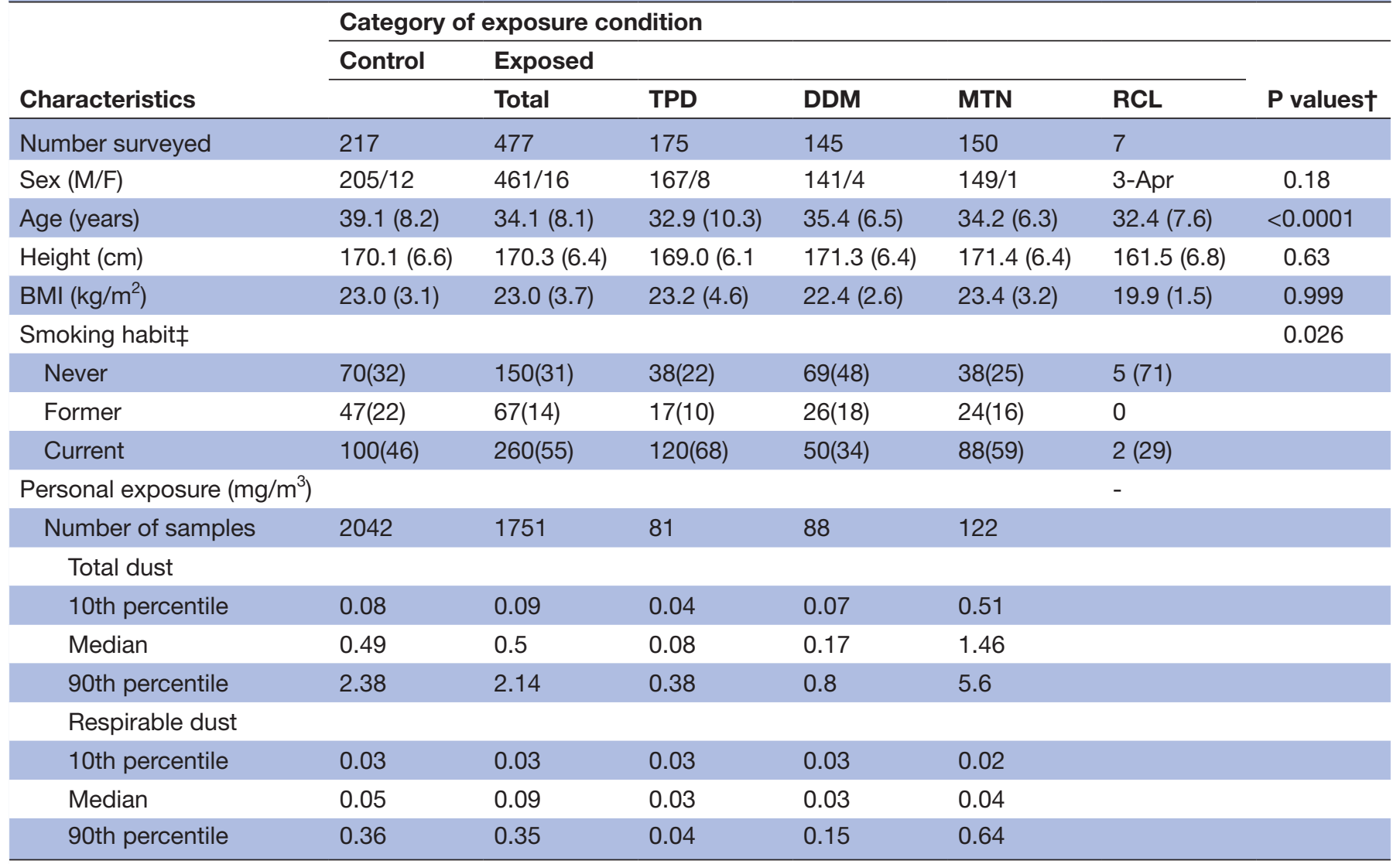

Values are presented as the number of subjects for sex and smoking habit and the arithmetic mean (SD) for age, height and BMI at the baseline survey.

†Probability under the hypothesis that there are no differences between the control group and the exposed group as a whole. †Percentage of column total in parentheses.

BMI, body mass index; DDM, machine design and development; MTN, maintenance; RCL, recycling; TPD, toner production.

Several background characteristics at the baseline point, as well as the results of personal exposure measurements during the entire study period as the 10th and 90th percentile values of the TWA-8h concentration, were compared among the exposure categories in table 1 . The male-tofemale ratio was comparable between the exposed and control groups. However, the absolute number of female subjects was quite small compared with males, with only 28 women analysed. Significant differences were observed between the exposed and control groups in mean age and smoking habit classification at the baseline survey, with the exposed group being younger and more likely to smoke than the controls. Seventy-six of 360 current smokers at baseline quit smoking during their follow-up period, whereas only 1 out of 220 never smokers at the point of baseline started smoking during the follow-up. As for body size, the mean values of standing height and body mass index (BMI) were comparable between the exposed and control groups.

The TPD and RCL workers tended to show higher percentile values both in the total and respirable TWA- $8 \mathrm{~h}$ than the DDM and MTN workers. As suggested by those summary percentile values, the individual TWA- $8 \mathrm{~h}$ values distributed across a rather wide range, and some of them exceeded the permissible limit recommended by JAIH $\left(4 \mathrm{mg} / \mathrm{m}^{3}\right.$ as total and $1 \mathrm{mg} / \mathrm{m}^{3}$ as respirable carbon black dust and $8 \mathrm{mg} / \mathrm{m}^{3}$ as total and $2 \mathrm{mg} / \mathrm{m}^{3}$ as respirable general dust). ${ }^{13}$ However, the overall median TWA-8h values of both total and respirable dust were well below the JAIH recommendations, even among the TPD and RCL workers. Figure 2 shows the time-dependent change in those summary statistics of personal exposure measurement during the study period. No marked trends were observed in the percentile values during the 10-year follow-up, suggesting that the work environment was relatively stable throughout the study period in terms of dust exposure to the workers as a whole.

Table 2 summarises the prevalence of the biomedical indices studied at the baseline survey according to the exposure categories. The ORs and their 95\% CIs of the exposed to the control group are also shown after adjustment for possible confounding variables. The exposed group tended to show higher prevalence in respiratory symptom indices, although significant differences were observed only in chronic phlegm and wheeze grade 2 . The prevalence values for CRP and IgE abnormalities were also significantly larger in the exposed group than in the control group, although the results of KL6 and SPD were 

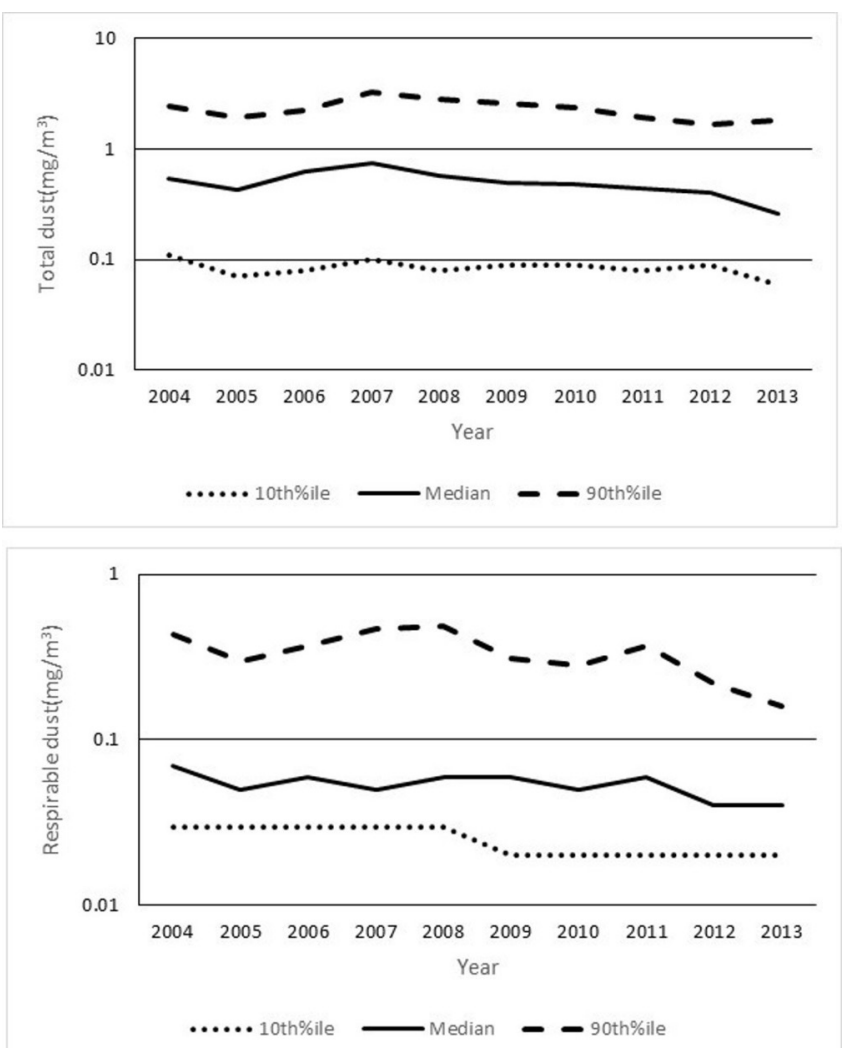

Figure 2 The exposure levels of toner-handling workers by year of measurement estimated by personal exposure measurements. The values are presented as the median and the 10th and 90th percentiles (\%ile) for personal exposure measurement data.

comparable between the two groups. The difference in CRP remained significant even after adjustment for BMI, a well-known contributor to the serum CRP level. Only a small number of subjects demonstrated values outside of the reference ranges of FVC, FEV1 or FEV1/FVC, and the prevalence showed values ranging from $2 \%$ to $6 \%$. There were no cases of clear pneumoconiosis with $1 / 1$ or a more severe grade on baseline chest X-ray films of the subjects analysed, although non-specific findings were noted in $3.4 \%$ of the exposed and $1.8 \%$ of the control subjects at baseline. No significant difference between the two groups was noted in the prevalence at baseline of either the pulmonary function or chest X-ray indices.

Table 3 compares the frequency of newly emerging abnormalities observed during the follow-up period between the exposed and control groups for selected biomedical indices in order to examine the degree of chronic respiratory deterioration, including lung fibrotic changes. This table also includes the results of comparisons according to work categories, although no RCL workers were included in this analysis because the number of such workers was too small, namely, only seven workers. After carefully evaluating the chest X-ray films accumulated during the follow-up period, we observed no cases with $1 / 1$ or a more severe grade of pneumoconiosis. In addition, the incidence of non-specific findings during the follow-up was comparable between the exposed and control groups. Furthermore, two clinically useful biomarkers of lung fibrosis (KL6 and SPD) showed rather low incidence values for newly emerging abnormalities, which were comparable between the exposed and control groups. However, regarding symptoms and the pulmonary function, the exposed group tended to have a higher incidence of abnormalities more frequently than the control group. The ORs estimated by logistic regression analyses mostly exceeded 1.5, and after adjustment for age and smoking status during the follow-up period, chronic cough emerged significantly more frequently in the exposed subjects than in the control group. In the analyses according to work categories, an increase in the OR value of chronic cough was observed in all exposed work categories that were analysed and reaching a statistically significant level in DDM and MTN workers. The TPD, the highest exposure category, tended to show a more consistent elevation of $\mathrm{OR}$ values versus the controls than other work categories.

Table 4 shows the comparison of FVC/ $\mathrm{HT}^{2}$ and FEV1/ $\mathrm{HT}^{2}$ decline during the follow-up period among the exposure categories. Comparable values were obtained for both indices between the exposed as a whole and the control groups. After adjustment for the significant contribution of age, toner exposure was not a significant factor influencing the decline in the pulmonary function. In the comparison among the three exposed work categories, the MTN workers showed a larger annual decline in both $\mathrm{FVC} / \mathrm{HT}^{2}$ and $\mathrm{FEV} 1 / \mathrm{HT}^{2}$ than TPD and DDM workers, although the differences were not statistically significant after adjusting for age.

Table 5 summarises the significant independent contribution of confounding factors in the multivariate analyses described in tables 2 and 3 as estimated ORs for the health-related outcomes. Only statistically significant results are presented with their 95\% CIs. Smoking was considered to increase the incidence of chronic phlegm, SPD abnormality and reduced FVC and FEV1 during follow-up, as well as the prevalence of many symptoms and a reduced pulmonary function at baseline. Similar increases in ORs with age were also observed for the incidence during the follow-up and the prevalence at baseline of some of the examined indices. A clear independent association between serum CRP abnormalities and obesity was also observed.

\section{DISCUSSION}

Photocopiers and laser printers using powdered toner are widely used in offices and homes, but the powdered toner for printing and emissions from these machines may become air pollutants in office and home environments. ${ }^{16}{ }^{17}$ McGarry et al reported the condition of particulate air pollution resulting from the use of laser printers in several actual office environments. ${ }^{18}$ As several case reports have suggested the existence of chronic pulmonary disorders possibly associated with inhaled toner 
Table 2 The comparison of the baseline status in select biomedical indices at the point of the first examination for each subject between the exposed subjects as a whole and the controls

\begin{tabular}{|c|c|c|c|c|c|}
\hline \multirow[b]{3}{*}{ Biomedical indices } & \multicolumn{2}{|c|}{ Univariate analysis } & \multirow[b]{3}{*}{ P values* } & \multicolumn{2}{|c|}{ Multivariate analysis } \\
\hline & \multicolumn{2}{|c|}{ Prevalence (\%) } & & \multicolumn{2}{|c|}{ Logistic regression model } \\
\hline & Exposed & Controls & & ORt & $95 \% \mathrm{Cl} \dagger$ \\
\hline \multicolumn{6}{|l|}{ Respiratory symptoms $\ddagger$} \\
\hline Cough \#1 (7A) & 19.4 & 14.8 & 0.14 & 1.35 & (0.85 to 2.14$)$ \\
\hline Cough \#2 (7B) & 14.8 & 11.1 & 0.19 & 1.41 & (0.84 to 2.37 ) \\
\hline Chronic cough (7E) & 5.5 & 4.2 & 0.46 & 1.6 & (0.70 to 3.64$)$ \\
\hline Phlegm \#1 (8A) & 23.7 & 20.4 & 0.34 & 1.23 & (0.81 to 1.87$)$ \\
\hline Phlegm \#2 (8B) & 13.5 & 10.7 & 0.29 & 1.45 & (0.85 to 2.48 ) \\
\hline Chronic phlegm (8E) & 8.9 & 4.2 & 0.03 & 2.62 & (1.20 to 5.70$)$ \\
\hline Exacerbation (9A) & 10.5 & 7 & 0.14 & 1.56 & (0.84 to 2.91$)$ \\
\hline Wheeze \#1 (10A1) & 9.5 & 5.6 & 0.09 & 1.51 & (0.76 to 2.99 ) \\
\hline Wheeze \#2§ (10A2) & 2.7 & 0 & 0.01 & - & - \\
\hline Chronic wheeze (10A3) & 0 & 0 & - & - & - \\
\hline Breathlessness \#1 (13A) & 9.7 & 11.6 & 0.45 & 1 & (0.58 to 1.73$)$ \\
\hline Breathlessness \#2§ (13B) & 2.2 & 1.4 & 0.76 & 2.27 & (0.58 to 8.89$)$ \\
\hline Breathlessness \#3§ (13C) & 0.4 & 0.9 & 0.59 & 0.96 & (0.13 to 7.20$)$ \\
\hline \multicolumn{6}{|l|}{ Biomarker abnormality } \\
\hline CRP & 12 & 6 & 0.02 & 2.02 & (1.05 to 3.88$)$ \\
\hline $\lg \mathrm{E}$ & 31.7 & 20.8 & 0.003 & 1.66 & (1.12 to 2.48$)$ \\
\hline 8OHdG§ & 1.1 & 1.8 & 0.47 & 0.68 & (0.17 to 2.78$)$ \\
\hline KL6§ & 0.4 & 0 & 1 & - & - \\
\hline SPD & 3.2 & 3.4 & 0.89 & 0.91 & (0.35 to 2.37$)$ \\
\hline \multicolumn{6}{|l|}{ Reduced pulmonary function } \\
\hline FVC & 6.5 & 6 & 0.76 & 1 & (0.49 to 2.00$)$ \\
\hline FEV1 & 6.1 & 2.8 & 0.06 & 2.09 & (0.83 to 5.27$)$ \\
\hline FEV1/FVC§ & 2.1 & 5.1 & 0.03 & 0.44 & (0.18 to 1.09$)$ \\
\hline \multicolumn{6}{|l|}{ Chest $\mathrm{X}$-ray findings } \\
\hline Fibrotic change $\geq 1 / 1$ & 0 & 0 & 1 & - & - \\
\hline Non-specific findings & 3.4 & 1.8 & 0.27 & 2.47 & (0.78 to 7.81$)$ \\
\hline
\end{tabular}

The figures in the column of prevalence represent the proportion of subjects with symptoms or positive findings in each index. The results of multivariate analyses were adjusted for the age and smoking status of the subjects. Body mass index was also used as an adjustment factor in the case of CRP.

*Probability under the hypothesis that there are no differences between the control group and exposed group as a whole examined by the $\chi^{2}$ test or Fisher's exact probability method.

TOR and its $95 \%$ Cls of the exposed group to the control group, regarding the positive findings at the baseline survey. $\ddagger$ The ATS-DLD-78A questionnaire code is shown in the parentheses.

§Fisher's exact probability method was applied.

\#Grade of symptom severity: cough \#1 and phlegm \#1: usually having the symptom; cough \#2 and phlegm \#2: 4-6 times a day on 4 or more days a week; wheeze \#1: when having a cold; wheeze \#2: occasionally apart from cold; breathlessness \#1: when hurrying on the level or walking up a slight hill; breathlessness \#2: having to walk slower than people your age on the level; breathlessness \#3: having to stop for breath when walking at your own pace.

8OHdG, 8-hydrovy deoxy guanosine; ATS, American Thoracic Society; CRP, C reactive protein; FEV1, forced expiratory volume in 1 s; FVC, forced vital capacity; IgE, immunoglobulin E; KL6, sialylated carbohydrate antigen KL-6; SPD, surfactant protein D.

dust, ${ }^{1-3}$ many aspects of toner-related health effects have been reported. Khatri et $a l^{19}$ conducted a human volunteer exposure study in an actual current office environment and found that several biomarkers, including $8 \mathrm{OHdG}$ as a marker of oxidative stress, ${ }^{20}$ changed in relation with the particulate air pollutants emitted by photocopiers and laser printers, although those changes were acute or subacute and not chronic in nature. Elango et $a l^{21}$ reported the result of a cross-sectional study on photocopier centre workers in India, showing the significant elevation of serum inflammatory biomarkers among the exposed workers compared with the controls. Kasi 


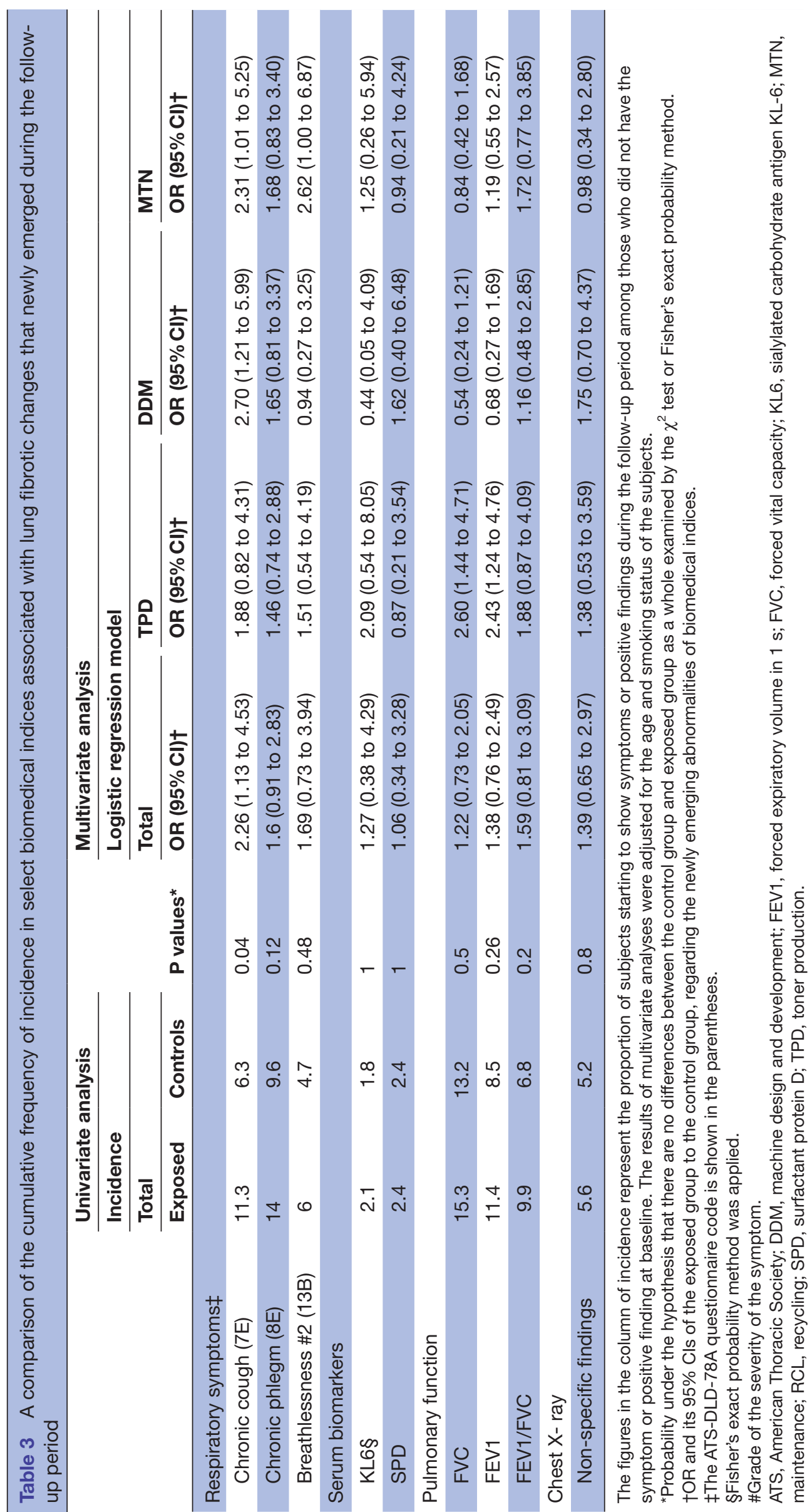


Table 4 Annual decline in spirometric indices according to exposure category

Category of exposure condition (number of subjects)

\begin{tabular}{|c|c|c|c|c|c|c|c|c|}
\hline \multirow[b]{3}{*}{ Spirometric indices } & \multirow{3}{*}{$\begin{array}{l}\text { Control } \\
(n=217)\end{array}$} & \multicolumn{5}{|l|}{ Exposed } & \multicolumn{2}{|c|}{$\begin{array}{l}\text { Statistical test of the } \\
\text { mean values* }\end{array}$} \\
\hline & & Total & TPD & DDM & MTN & $\mathrm{RCL}$ & $P$ values & \\
\hline & & $(n=477)$ & $(n=175)$ & $(n=145)$ & $(n=150)$ & $(n=7)$ & Unadjusted & Adjusted $\dagger$ \\
\hline$\Delta \mathrm{FVC} / \mathrm{HT}^{2}\left(\mathrm{~mL} / \mathrm{m}^{2}\right)$ & $-6.2(21.4)$ & $-4.0(19.1)$ & $-0.7(25.8)$ & $-2.5(12.5)$ & $-9.0(14.3)$ & $-5.8(13.3)$ & 0.19 & 0.5 \\
\hline$\Delta \mathrm{FEV} 1 / \mathrm{HT}^{2}\left(\mathrm{~mL} / \mathrm{m}^{2}\right)$ & $-8.8(16.3)$ & $-7.5(13.9)$ & $-6.5(16.3)$ & $-7.3(10.7)$ & $-9.0(12.5)$ & $-6.7(32.6)$ & 0.33 & 0.3 \\
\hline
\end{tabular}

Values are the arithmetic means and SD at the point of baseline survey.

$\dagger^{\star}$ Comparison between the control and the exposed as a whole.

†Adjusted for age, sex and smoking habit at the baseline.

DDM, machine design and development; FEV1, forced expiratory volume in $1 \mathrm{~s}$; FVC, forced vital capacity; $\mathrm{H}^{2}$, height-squared; MTN,

maintenance; RCL, recycling; TPD, toner production.

et $a l^{22}$ suggested possible genotoxicity associated with photocopier-related exposure in a survey of photocopier operators and maintenance workers. Yanagi $e t a l^{23}$ found no marked difference in the pulmonary function due to toner-related work in their cross-sectional analysis of workers at a business equipment manufacturer. In their critical review, Pirela $e t a l^{24}$ stated that particulate matter, including nanoparticles emitted from photocopiers and laser printers during operations, could exert biological effects on the respiratory system, such as oxidative stress and inflammatory responses.

Compared with human volunteer or cross-sectional epidemiological studies, the evidence obtained by longitudinal epidemiological studies is quite limited, but longitudinal study results are needed to fully examine the health impact of particulate air pollutants associated

Table 5 Independent contribution of confounding factors in the multivariate analyses of biomedical indices

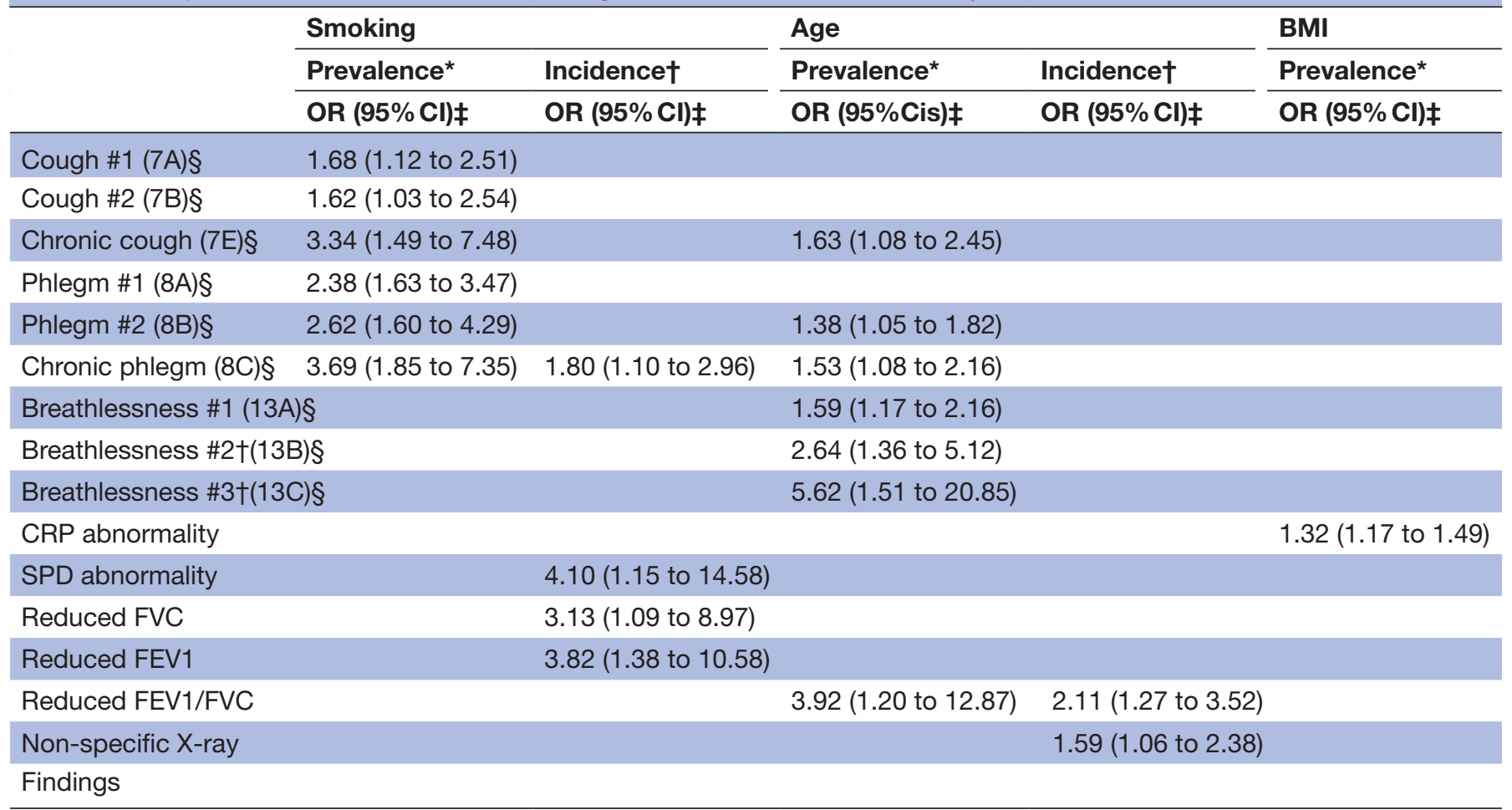

Values are presented as the OR $(95 \% \mathrm{Cl})$ estimated using the logistic regression models shown in tables 3 and 4 . Only statistically significant results are shown.

${ }^{*}$ An analysis of the prevalence at baseline.

†An analysis of the new incidence during the follow-up observation.

$\ddagger \mathrm{OR}$ and its $95 \% \mathrm{Cl}$ of the exposed group to the control group, regarding the prevalence and incidence of biomedical indices. $\S$ The ATS-DLD-78A questionnaire code is shown in the parentheses.

\#Grade of the severity of the symptom.

ATS, American Thoracic Society; CRP, C reactive protein; FEV1, forced expiratory volume in $1 \mathrm{~s}$; FVC, forced vital capacity; SPD, surfactant protein D. 
with office machines, as far as chronic health effects are concerned. In a cohort study on toner and photocopier manufacturing workers conducted over 4years, no evidence was obtained regarding the adverse effects on the pulmonary function or chest X-ray findings associated with toner-handling work, ${ }^{25} 26$ although an increased prevalence of breathlessness was observed in association with toner-handling work. ${ }^{27}$ However, those studies did not evaluate the incidence of abnormalities based on longitudinally observed data. Therefore, we conducted a longitudinal study to further evaluate the possible health effects of toner dust exposure as a subsequent study of our cross-sectional one mentioned above ${ }^{4}$ with a focus mainly on the chronic respiratory effects, such as lung fibrotic changes. To determine the actual risk in the current realworld environment, we studied an actual working population. Personal exposure measurement results showed that the current level of exposure to total and respirable dust in toner-handling workers was on average well below the permissible exposure levels of general mineral dust and carbon black dust recommended by $\mathrm{JAIH},{ }^{13}$ even among TPD and RCL workers, in whom relatively high exposure was observed. We also used several biomedical indices to detect possible harmful effects on the respiratory system: namely imaging findings by chest X-ray, KL6 and SPD levels in serum as clinically used biomarkers on lung fibrosis, spirometry results to evaluate the lung functional status and subjective symptoms obtained through standardised methods.

In a cross-sectional analysis of the baseline results, the exposed group tended to have subjective symptoms more frequently than in the control group, and similar group differences were observed in the frequency of abnormal values in the majority of the biomedical indices examined. In addition, the difference between the two groups reached a statistically significant level in several items, even after controlling for confounders. This tendency was basically consistent with what we had previously reported, with exposed workers complaining of symptoms more frequently than control workers despite having a comparable frequency of pulmonary function and chest radiography abnormalities. Although the presence of consistent difference between the groups might suggest the existence of certain subclinical changes, the cross-sectional evaluation at the baseline might have been influenced by a number of factors prior to the start of this study. Therefore, newly emerging abnormalities during follow-up should be examined in subjects without any abnormalities at the baseline observation, as far as the chronic health effects are concerned. One interesting finding of the cross-sectional analysis was the higher prevalence of CRP abnormalities, a sensitive marker of acute inflammatory status in the body, among the exposed subjects compared with control subjects. It seems unlikely that the elevated CRP values were significantly influenced by events in the past due to its nature as an acute inflammatory biomarker. In addition, the difference seemed to be independent of inflammatory conditions caused by obesity or smoking, as both were used as adjustment factors in the logistic regression model. These findings suggest that acute inflammatory conditions might be associated with current toner exposure. At the same time, an increase in the serum IgE level was also frequently found in the exposed subjects. In Japan, an allergy to cedar pollen is commonly observed every spring, so cedar pollenosis may be related to the findings for CRP and IgE. However, as there are multiple factors affecting the $\mathrm{CRP}^{28}$ as well as the IgE levels, we were unable to specify the reason for that group difference.

In the longitudinal analyses of biomedical indices, we evaluated the frequency of a newly emerging abnormality during the follow-up period by excluding the subjects from the analysis if they already had the finding at baseline. Among the indices associated with lung fibrotic changes, we found closely similar results between the exposed and control groups regarding the new incidence of chest radiographic findings and serum biomarker abnormalities. These results suggest that the fibrogenic potential of dust exposure associated with toner-handling work seems to be minimal, if any at all, as far as the current working environment is concerned.

In contrast, the estimated ORs for FEV1/FVC abnormality adjusted for confounders showed a relatively a large value exceeding 1.5, although the increase was not statistically significant. A statistically significant increase in the OR of TPD versus the controls was observed in FVC and FEV1 abnormalities. However, the overall differences in the incidence of reduced FVC and FEV1 were relatively small between the exposed subjects and control subjects. Furthermore, the annual declines in the FVC/ $\mathrm{HT}^{2}$ and FEV1/ $\mathrm{HT}^{2}$ of the exposed subjects during the follow-up period were comparable with those of the control subjects after controlling for the age and smoking status during the follow-up. Thus, it seems difficult to attribute certain biological or medical reasons to the moderate but a insignificant elevation in ORs that was seen regarding the pulmonary function index abnormality. Some fluctuation around normal ranges might be partially responsible for the observed elevation in OR values. In the case of chronic cough, the exposed group showed a larger incidence than the control group. This finding was not associated with the findings for chronic phlegm, which showed a comparable incidence after adjusting for the significant contribution of smoking. This suggests that the significant increase in the rate of chronic cough cannot be attributed to mucus hypersecretion. Given the findings at baseline mentioned earlier, it might be possible to hypothesise that non-specific irritation leading to subjective symptoms, fluctuation in the pulmonary function and inflammatory response were caused to some extent by exposure associated with toner-handling work.

However, of note: in all indices selected for the longitudinal incidence analyses, the OR value adjusted for confounders exceeded the unit value without exception, suggesting mostly non-significant but consistent elevation of the incidence in the exposed workers. Therefore, 
further epidemiological studies are needed in order to identify mild and non-specific effects in a large-scale sample with sufficient statistical power. In this context, a similar tendency towards a consistent OR elevation was observed in TPD work, which was associated with the greatest exposure of total and respirable dust in the personal exposure measurement. In the comparison of the pulmonary function decline, the largest annual losses of $\mathrm{FVC} / \mathrm{HT}^{2}$ and $\mathrm{FEV} 1 / \mathrm{HT}^{2}$ were seen in the MTN category, where the degree of dust exposure was smaller than that for TPD. However, it was difficult to perform a detailed analysis by the work exposure categories with regard to the degree of toner exposures, as no information was obtained regarding the exact percentage of toner included in the total dust sampled. The toner particle exposure should be measured separately from other substances in future studies in order to enable detailed analyses of the dose-response relationship as well as to facilitate focusing on specific work categories, such as TPD and MTN.

Several limitations associated with the present study warrant mention. First, the number of subjects of this study (694 in total) might be insufficient to detect minimal and subclinical health deteriorations, although this study seems to have reasonably sufficient power to detect at least well-established health risks, such as ageing, smoking and obesity. A meta-analysis of the studies with a common methodological basis will be useful for detecting minimal effects of toner dust. Second, we obtained measurements on personal exposure concentration for each work category as an exposure assessment. However, the results showed large variability. In addition, we were unable to obtain reliable data on the relative content of toner as a fraction of the whole dust sample. We therefore cannot reliably estimate the amount of personal exposure to toner dust for each individual subject. An analysis of the dose-response relationship will require a more precise exposure assessment for toner dust. Third, we simply used conventional chest radiography and did not use CT to evaluate lung fibrotic changes, because we should minimise the amount of X-rays that the participants were exposed to in an epidemiological research setting. We were therefore unable to distinguish the non-specific findings in cases where extremely early phase of toner-related changes might have been included. Advanced radiography techniques will be useful for the more precise evaluation of lung fibrotic deterioration caused by toner dust inhalation.

\section{CONCLUSION}

Under the current reasonably controlled work environmental conditions, lung fibrotic changes caused by inhaled dust exposure, including powdered toner, appear to be relatively uncommon; however, non-specific temporal irritation causing subjective symptoms and inflammatory responses might exist. Further epidemiological research will be useful for clarifying the possible health effects of toner dust exposure fully, especially the minimal and non-specific health changes in a study setting with a sufficient sample size to detect these changes.

Acknowledgements The authors would like to thank all the subjects, investigators and survey staff who participated in this longitudinal study over 10 years.

Contributors TN designed the study; TN and YY executed the data collection in annual surveys; YY, TY, SO and DN contributed to the data preparation and analyses; TN wrote the initial manuscript. All of the authors contributed to the revision of the manuscript and approved the final form of the manuscript for submission.

Funding This study was funded in part by Ricoh Co, Ltd, a photocopier industry company in Japan.

Competing interests None declared.

Patient consent Not required.

Ethics approval This study was conducted under the approval of the Institutional Review Board on Medical Ethics of Showa University School of Medicine (Authorisation No. 201).

Provenance and peer review Not commissioned; externally peer reviewed. Data sharing statement No additional data available.

Open access This is an open access article distributed in accordance with the Creative Commons Attribution Non Commercial (CC BY-NC 4.0) license, which permits others to distribute, remix, adapt, build upon this work non-commercially, and license their derivative works on different terms, provided the original work is properly cited, appropriate credit is given, any changes made indicated, and the use is non-commercial. See: http://creativecommons.org/licenses/by-nc/4.0/.

\section{REFERENCES}

1. Gallardo M, Romero P, Sánchez-Quevedo MC, et al. Siderosilicosis due to photocopier toner dust. Lancet 1994;344:412-3.

2. Armbruster C, Dekan G, Hovorka A. Granulomatous pneumonitis and mediastinal lymphadenopathy due to photocopier toner dust. Lancet 1996;348:690.

3. Wieriks J. Photocopier toner dust and lung disease. Lancet 1996;348:1518-9.

4. Nakadate T, Yamano $\mathrm{Y}$, Adachi $\mathrm{C}$, et al. A cross sectional study of the respiratory health of workers handling printing toner dust. Occup Environ Med 2006;63:244-9.

5. Department of Health and SafetyMinistry of Labour, Japan. Jinpai Shinsa handbook (Pneumoconiosis examination handbook. Tokyo: Japan Safety and Health Association, 1978.

6. Ministry of Labour, Japan. Jinpai Hyoujun $x$-ray films (Standard x-ray films for diagnosis of pneumoconiosis. Tokyo: Japan Safety and Health Association, 1982.

7. International Labour Organization. International Classification of Radiographs of Pneumoconioses, 2002.

8. Ferris BG. Epidemiology standardization project (American Thoracic Society). Am Rev Respir Dis 1978;118:1-120.

9. Standardization of spirometry, 1994 update. American Thoracic Society. Am J Respir Crit Care Med 1995;152:1107-36.

10. Nakadate T, Aizawa Y, Yagami T, et al. Change in obstructive pulmonary function as a result of cumulative exposure to welding fumes as determined by magnetopneumography in Japanese arc welders. Occup Environ Med 1998;55:673-7.

11. Kubota M, Kobayashi H, Quanjer PH, et al. Reference values for spirometry, including vital capacity, in Japanese adults calculated with the LMS method and compared with previous values. Respir Investig 2014;52:242-50.

12. Nakadate T. Longitudinal observation of pulmonary function decline in male Japanese workers: design, baseline results, and methodological considerations of handling pulmonary function data. Ind Health 1998;36:305-11.

13. Japan association of industrial health. recommendation on threshold limit values, Japan (2016). Sangyo Eiseigaku Zasshi 2016;58:181-212.

14. Hermans $C$, Bernard A. Lung epithelium-specific proteins: characteristics and potential applications as markers. Am J Respir Crit Care Med 1999;159:646-78.

15. Ministry of Education, Culture, Sports, Science and Technology, and Ministry of Health, Welfare, and Work, Japan. Ethical Guidelines for Epidemiological Studies. http://www.mhlw.go.jp/general/seido/ kousei/i-kenkyu/ekigaku/0504sisin.html. 
16. He C, Morawska L, Taplin L. Particle emission characteristics of office printers. Environ Sci Technol 2007;41:6039-45.

17. Tang T, Hurraß J, Gminski R, et al. Fine and ultrafine particles emitted from laser printers as indoor air contaminants in German offices. Environ Sci Pollut Res Int 2012;19:3840-9.

18. McGarry P, Morawska L, He C, et al. Exposure to particles from laser printers operating within office workplaces. Environ Sci Technol 2011;45:6444-52.

19. Khatri M, Bello D, Gaines P, et al. Nanoparticles from photocopiers induce oxidative stress and upper respiratory tract inflammation in healthy volunteers. Nanotoxicology 2013;7:1014-27.

20. Irie M, Tamae K, Iwamoto-Tanaka N, et al. Occupational and lifestyle factors and urinary 8-hydroxydeoxyguanosine. Cancer Sci 2005;96:600-6.

21. Elango N, Kasi V, Vembhu B, et al. Chronic exposure to emissions from photocopiers in copy shops causes oxidative stress and systematic inflammation among photocopier operators in India. Environ Health 2013;12:78.

22. Kasi V, Elango N, Ananth S, et al. Occupational exposure to photocopiers and their toners cause genotoxicity. Hum Exp Toxicol 2018;37:205-17.
23. Yanagi N, Kitamura H, Mizuno M, et al. A 4-year follow-up cohort study of the respiratory functions in toner-handling workers. Saf Health Work 2014;5:222-6.

24. Pirela SV, Martin J, Bello D, et al. Nanoparticle exposures from nano-enabled toner-based printing equipment and human health: state of science and future research needs. Crit Rev Toxicol 2017;47:683-709.

25. Kitamura $\mathrm{H}$, Terunuma N, Kurosaki S, et al. A cohort study using pulmonary function tests and $\mathrm{x}$-ray examination in toner-handling workers: cross-sectional and longitudinal analyses from 2003 to 2008. Hum Exp Toxicol 2015;34:345-56.

26. Ikegami K, Hasegawa $\mathrm{M}$, Ando $\mathrm{H}$, et al. A cohort study of the acute and chronic respiratory effects of toner exposure among handlers: a longitudinal analyses from 2004 to 2013. Ind Health 2016;54:448-59.

27. Kitamura $\mathrm{H}$, Terunuma N, Kurosaki S, et al. A cohort study on selfreported respiratory symptoms of toner-handling workers: crosssectional and longitudinal analysis from 2003 to 2008. Biomed Res Int 2014;2014:1-10.

28. Landry A, Docherty P, Ouellette S, et al. Causes and outcomes of markedly elevated C-reactive protein levels. Can Fam Physician 2017;63:e316-e323. 